\title{
Successful production of genome-edited rats by the rGONAD method
}

\author{
Tomoe Kobayashi $^{1 \dagger}$, Masumi Namba ${ }^{1 \dagger}$, Takayuki Koyano $^{1 \dagger}$, Masaki Fukushima ${ }^{1,2}$, Masahiro Sato ${ }^{3}$, \\ Masato Ohtsuka ${ }^{4,5,6}$ and Makoto Matsuyama ${ }^{1 *}$
}

\begin{abstract}
Background: Recent progress in development of the CRISPR/Cas9 system has been shown to be an efficient gene-editing technology in various organisms. We recently developed a novel method called Genome-editing via Oviductal Nucleic Acids Delivery (GONAD) in mice; a novel in vivo genome editing system that does not require ex vivo handling of embryos, and this technology is newly developed and renamed as "improved GONAD" (i-GONAD). However, this technology has been limited only to mice. Therefore in this study, we challenge to apply this technology to rats.

Results: Here, we determine the most suitable condition for in vivo gene delivery towards rat preimplantation embryos using tetramethylrhodamine-labelled dextran, termed as Rat improved GONAD (rGONAD). Then, to investigate whether this method is feasible to generate genome-edited rats by delivery of CRISPR/Cas9 components, the tyrosinase (Tyr) gene was used as a target. Some pups showed albino-colored coat, indicating disruption of wild-type Tyr gene allele. Furthermore, we confirm that rGONAD method can be used to introduce genetic changes in rat genome by the ssODN-based knock-in.

Conclusions: We first establish the rGONAD method for generating genome-edited rats. We demonstrate high efficiency of the rGONAD method to produce knock-out and knock-in rats, which will facilitate the production of rat genome engineering experiment. The rGONAD method can also be readily applicable in mammals such as guinea pig, hamster, cow, pig, and other mammals.
\end{abstract}

Keywords: CRISPR/Cas9, i-GONAD, rGONAD, Rat, Knock-out, Knock-in, In vivo electroporation

\section{Background}

The laboratory rat (Rattus norvegicus) has long been used in many studies to model a specified trait of human diseases (as exemplified by hypertension, diabetes and renal diseases), and for testing the drugs [1-4]. For example, Wistar Kyoto (WKY) strain is known to be uniquely susceptible to crescentic glomerulonephritis among the rat strains tested [5, 6]. Injection of isologous monoclonal antibodies caused anti-glomerular basement membrane antibody-induced glomerulonephritis (anti-GBM nephritis) in WKY rats [7, 8]. Notably, there is no mouse strain showing anti-GBM nephritis induced by such types of monoclonal antibodies.

\footnotetext{
* Correspondence: matsuyama@shigei.or.jp

${ }^{\dagger}$ Equal contributors

${ }^{1}$ Division of Molecular Genetics, Shigei Medical Research Institute, 2117

Yamada, Minami-ku, Okayama 701-0202, Japan

Full list of author information is available at the end of the article
}

However, despite the usefulness/importance of rats as experimental animals, production of genetically engineered rats has yet not been extensively proceeded during the past decades. Gene targeting using mouse ES cells used as an invaluable tool for exploring gene functions in mammals [9], however, these advantages are restricted in limits of the mouse model.

Establishment of successful rat ES cell culture was just made in 2010 [10]. In 2011 to 2013, a more convenient and simpler technology, called genome-editing technology as exemplified by zinc finger nucleases (ZFN) [11, 12], transcription activator-like effector nucleases (TALEN) [13], and CRISPR/Cas9 [14-16] capable of modifying a specific gene function in mammals appeared. With these technologies, it becomes possible to create genetically modified rats in a more convenient manner than ever. 
The recent CRISPR/Cas9 system is the simplest for generating animals carrying a modified genome. The system consists of Clustered Regularly Interspaced Short Palindromic Repeats (CRISPR) RNA and CRISPRassociated (Cas) nuclease protein $[17,18]$. This system is efficiently generated with knock-out and knock-in animals at targeted sequences [19, 20]. The CRISPR/Cas9 system simplifies the procedure for producing mutant animals, however, genome engineering in rat is still difficult task in most laboratories [21].

The most widely employed procedures for creating genome-edited animals include 3 major ex vivo embryo handling steps $[15,16,19]$ : namely 1 ) isolation of zygotes from a pregnant female that were previously mated with a male, 2) zygote microinjection of genome editing components, and 3) surgical transfer of microinjected zygotes into oviduct of a pseudopregnant female. These 3 steps require very high level of technical expertise and its proficiency of the researchers/technicians to perform these procedures, and expensive apparatus such as micromanipulator.

To simplify these complexed and more laborious processes, Ohtsuka and their colleagues developed a novel genome engineering method, called Genome-editing via Oviductal Nucleic Acids Delivery (GONAD) in mice [22-24]. This method involves in the in vivo genomeediting towards early preimplantation embryos present in an oviduct of pregnant females. Therefore, it does not require above-mentioned ex vivo handling of embryos, such as isolation of zygotes, zygote microinjection and transfer of the injected embryos to recipient females. In our first GONAD attempt using Cas9 mRNA and sgRNA, genome editing efficiency was about 25\% [22]. It was subsequently improved up to nearly $100 \%$ by using Cas9 protein and crRNA/tracrRNA complex (improved GONAD; $i$-GONAD) [24]. Although $i$-GONAD method is possibly applied to other organisms, unfortunately, this technology has been limited only to mice.

In this study, we attempted to apply this technology to rats. For this purpose, we used pigmented DA and albino WKY rat strains. We here examined whether 1) CRISPR/Cas9-mediated induction of indel is possible towards the wild-type tyrosinase (Tyr) locus, and 2) CRISPR/Cas9-mediated knock-in (KI) of single stranded (ss) oligonucleotides (ODN) in the mutated Tyr is possible by using the rGONAD method.

\section{Results}

\section{Determination of optimal electroporation efficiency for rGONAD}

In our mouse $i$-GONAD method, the electroporation was performed at 0.7 day of pregnancy (E0.7), at which embryonic stage corresponds to late 1-cell mouse embryos [24]. In the present study with rats, it was confirmed that over $80 \%$ of embryos collected from females (both with or without super-ovulation) at 4:00 pm were still 1-cell stage (E0.75) (data not shown). Next, to investigate the optimal condition of electroporation for effective introduction of CRISPR/Cas9 reagents into rat embryos, we examined tetramethylrhodamine-labelled dextran $(3 \mathrm{kDa})$ solution as a fluorescent indicator to evaluate $i$-GOAND method. We instilled 2-2.5 $\mu \mathrm{l}$ solution containing tetramethylrhodamine-labelled dextran $(0.5 \mu \mathrm{g} / \mu \mathrm{l})$ into oviductal lumen of super-ovulated pregnant WKY female rats at E0.75 (Fig. 1a-c, Additional file 1: movie S1) and then electroporated them using a NEPA21 electroporator as described in the Methods section (Fig. 1d-f, Additional file 2: movie S2). We designed 5 different conditions of electroporation (voltage with poring pulse: $50 \mathrm{~V}$, or $40 \mathrm{~V}$, or $30 \mathrm{~V}$; number of pulses with transfer pulse: 3 times or 6 times; see Fig. $1 \mathrm{f}$, i) in WKY rat embryos. Two days after in vivo electroporation, the embryos were isolated from the treated females and examined presence/absence of fluorescence. In the embryos with successful delivery of the dextran, some embryos exhibited distinct tetramethylrhodaminederived red fluorescence in their cytoplasm (Fig. 1g, h). As shown in Fig. 1i and Table 1, the 6 times of transfer pulse is highly efficient to the electroporation compared with those of 3 times. To investigate the differences of the electroporation efficiency in other rat stains, DA and WKY/DA F1 rat embryos were performed. In all 3 rat strains, there was no detectable difference in the delivery efficiency among stains (Fig. 1i-k, Table 1). These data suggest that "voltage with a poring pulse: $50 \mathrm{~V}$, and number of pulses with transfer pulse: 6 times" in the most suitable condition in rats. We termed this GONAD method as Rat improved GONAD (rGONAD).

\section{rGONAD-based gene disruption (KO)}

Then, to investigate whether rGONAD is feasible to generate genome-edited rats by delivery of CRISPR/Cas9 components, tyrosinase (Tyr) gene, encoding the enzyme responsible for the melanin synthesis, was used as a target of this experiment as previously reported [25]. The gRNA targeted to the wild-type Tyr allele was used to test the allele-specific genome-editing. Cas9 protein and gRNA were instilled into the oviducts of E0.75 WKY and DA female rats containing the fertilized eggs with hybrid genotypes (WKY x DA, F1), and electroporation was performed (Fig. 2a). The conditions of electroporation were the voltage with poring pulse: $50 \mathrm{~V}$, or $40 \mathrm{~V}$, or $30 \mathrm{~V}$; number of pulses with transfer pulse: 6 times.

Unfortunately, we could hardly obtain pups from superovulated female rats in our initial trial (WKY: injected females; 12, pregnant females; 2; DA: injected females; 14 , pregnant females; 0 ). Therefore, we decided to use the estrous female rats without super-ovulation for the following study. Such females became successfully pregnant and 

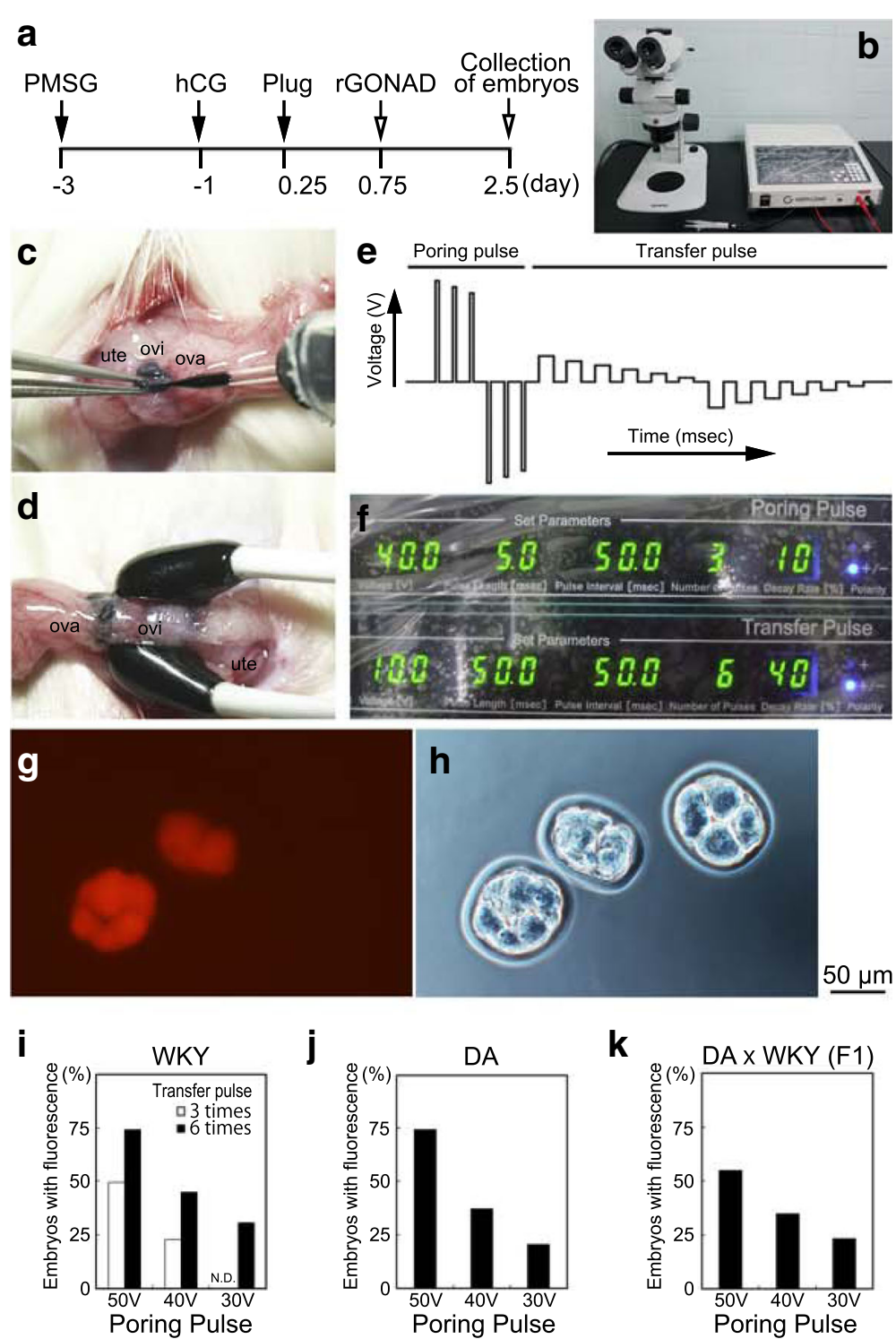

Fig. 1 Determination of optimal electroporation efficiency for rGONAD. a Scheme of experimental procedures for evaluation of electroporation efficiency using GONAD method. b SZX7 stereomicroscope and Super Electroporator NEPA21. c Tetramethylrhodamine-labelled dextran is instilled into the oviduct lumen using a micropipette inserted through the oviductal wall near the infundibulum. $\mathbf{d}$ After the injection, the oviductal regions were covered with a piece of wet paper, and then, electroporation is performed using tweezer-type electrodes. e, $\mathbf{f}$ Scheme (e) or diagram (f) of electroporation conditions delivered of by an electroporator. This electroporation parameters were; Poring pulse; $40 \mathrm{~V}, 5 \mathrm{msec}$ pulse, $50 \mathrm{msec}$ pulse interval, number of pulse 3 times, $10 \%$ decay ( \pm pulse orientation) and Transfer pulse; $10 \mathrm{~V}, 50 \mathrm{msec}$ pulse, $50 \mathrm{msec}$ pulse, number of pulse 6 times, $40 \%$ decay ( \pm pulse orientation). $\mathbf{g}, \mathbf{h}$ Fluorescence analysis of Tetramethylrhodamine-labelled dextran using GONAD method. $\mathbf{i}-\mathbf{k}$ Graph shows analysis of the percentage of electroporation efficiency in WKY (i), DA (j), and DA x WKY (k). Ova, ovary; Ovi, Oviduct; Ute, uterus. Scale bars: $50 \mu \mathrm{m}(\mathbf{g}, \mathbf{h})$

delivered pups (Table 2, Additional file 3: Table S1). Some pups showed albino-colored coat, indicating disruption of wild-type Tyr gene allele (Fig. 2b). Sequence analyses of their genomic DNA indicated that all the pups showing albino phenotype carried indel mutations at the target region of the Tyr gene (Fig. 2c, d). Of the pups that were electroporated with 50,40 , and $30 \mathrm{~V}$ of the voltage, 58.7, 35.3, and 13.4\%, the pups had edited Tyr gene, respectively (Table 2, Fig. 2e, Additional file 3: Table S1). No significant difference was detected in the gene editing efficiency between the strains of female rats; WKY and DA (Table 2, Fig. 2f, Additional file 3: Table S2), suggesting that $r$ GONAD is adaptable for all rat strains.

In addition, to study possible germ-line transmission of mutated alleles in rats obtained by the rGONAD, we obtained several F1 offspring by crossing between F0 founders and wild type rats. The germline transmission was confirmed in the next generations (Additional file 3: 
Table 1 Fluorescence ratio of teratmethlrhodamin-labelled dextran in rat embryos

\begin{tabular}{|c|c|c|c|c|c|}
\hline Stain & $\begin{array}{l}\text { Poring Pulse } \\
\text { Voltage }(V)\end{array}$ & $\begin{array}{l}\text { Transfer pulse } \\
\text { No. of Pulses }\end{array}$ & $\begin{array}{l}\text { No. of }>2 \text {-cell } \\
\text { embryos (A) }\end{array}$ & $\begin{array}{l}\text { No. of embryos with } \\
\text { fluorescence (B) }\end{array}$ & $\%(B / A)$ \\
\hline \multirow[t]{5}{*}{ WKY } & 50 & 3 & 138 & 68 & 49.3 \\
\hline & 50 & 6 & 116 & 86 & 74.1 \\
\hline & 40 & 3 & 158 & 36 & 22.8 \\
\hline & 40 & 6 & 124 & 56 & 45.2 \\
\hline & 30 & 6 & 123 & 38 & 30.9 \\
\hline \multirow[t]{3}{*}{ DA } & 50 & 6 & 118 & 88 & 74.6 \\
\hline & 40 & 6 & 109 & 41 & 37.6 \\
\hline & 30 & 6 & 110 & 23 & 20.9 \\
\hline \multirow[t]{3}{*}{$W K Y \times D A(F 1)$} & 50 & 6 & 122 & 67 & 54.9 \\
\hline & 40 & 6 & 154 & 54 & 35.1 \\
\hline & 30 & 6 & 133 & 31 & 23.3 \\
\hline
\end{tabular}

Table S3). These results indicate that it is possible to create genome-edited rat lines by the rGONAD method.

\section{Recovery of coat-color mutation using ssODN-based knock-in (KI) approach}

To analyze whether rGONAD method can be used to introduce genetic changes in rat genome, we next examined to repair mutation associated with representative recessive coat-color phenotypes in rat, as previous report [25]. To recover the albino phenotype caused by the point mutation, we designed a gRNA for a region spanning the mutation and a 100-bases long ssODN repair template that corresponds to the wild-type sequence of Tyr gene (Fig. 3a). These components were instilled into oviducts of pregnant WKY rats and subjected to electroporation. As a result, some of the pups showed albino with non-agouti, hooded phenotype, indicating successful correction of Tyr gene mutation (Fig. 3b). Sequence analyses of genomic DNA demonstrated that the pups showing pigmentation phenotype contained the corrected Tyr sequence (Fig. 3c). Of the pups that obtained from females that underwent electroporation with 50 and $40 \mathrm{~V}$ poring pulse, 50.0 and
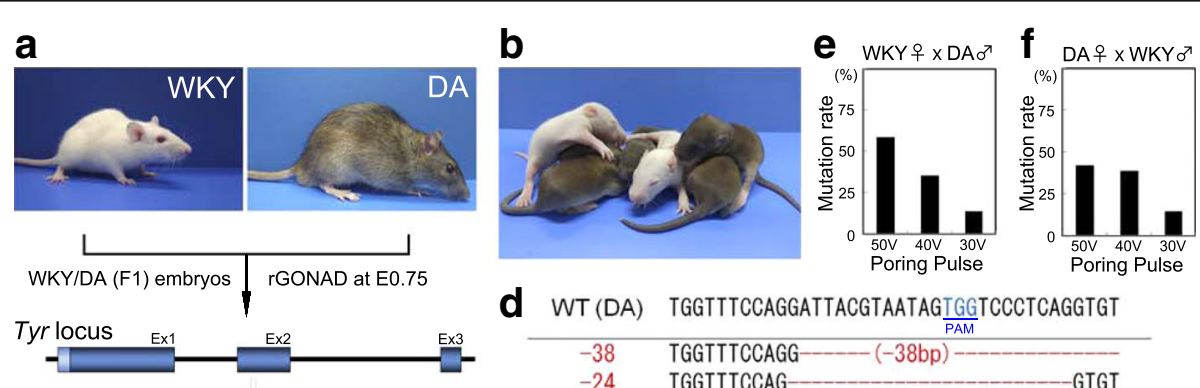

d WT(DA) tgGtTtccaggattacgtaAtagtgGtCCCTCAggtgt

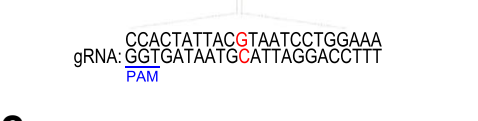

\begin{tabular}{|c|c|}
\hline-38 & $-(-38 \mathrm{bn})-\ldots$ \\
\hline-24 & TGGTTTCCAG- \\
\hline-14 & TGGTTTCCA- - TGGTCCCTCAGGTGT \\
\hline-12 & TGGTTTCCAG-_-_GTGGTCCCTCAGGTGT \\
\hline-9 & TGGTTTCCAGGATTACGT_—CCCTCAGGTGT \\
\hline$-7+1$ & TGGTTTCCAGGATTA--T-_-GTGGTCCCTCAGGTGT \\
\hline-7 & TGGTTTCCAGGAT- - - TAGTGGTCCCTCAGGTGT \\
\hline$-4+1$ & TGGTTTCCAGGATTACGTAA--A-GGTCCCTCAGGTGT \\
\hline-3 & TGGTTTCCAGGATTACGTA--GTGGTCCCTCAGGTGT \\
\hline-3 & TGGTTTCCAGGATTACG---TAGTGGTCCCTCAGGTGT \\
\hline-1 & TGGTTTCCAGGATTACGT-ATAGTGGTCCCTCAGGTGT \\
\hline$C \rightarrow T$ & TGGTTTCCAGGATTATGTAATAGTGGTCCCTCAGGTGT \\
\hline+1 & TGGTTTCCAGGATTACGTAATTAGTGGTCCCTCAGGTGT \\
\hline+1 & TGGTTTCCAGGATTACGTAAGTAGTGGTCCCTCAGGTGT \\
\hline+1 & TGGTTTCCAGGATTACGTAAATAGTGGTCCCTCAGGTGT \\
\hline+2 & TGGTTTCCAGGATTACGTAAAATAGTGGTCCCTCAGGTGT \\
\hline
\end{tabular}

Fig. 2 Production of Tyr knock-out (KO) rats by the rGONAD method. a Scheme of allele-specific genome editing for knock-out rats. The rGONAD method was performed in 0.75 day pregnant albino WKY crossed to agouti DA strains, which the fertilized eggs are (WKY $x$ DA) F1 hybrid. The target sequence and PAM at Tyr locus are shown. $\mathbf{b}$ Some the editing rats had albino colored coats. $\mathbf{c}$ Direct sequencing results of wild-type F1 (upper; WT) or the editing (below; indel) rats. Red arrow indicates indel mutaion. $\mathbf{d}$ Sequence analysis of the pups showed a variety of indel mutation at the Tyr locus, as shown in red. e, $\mathbf{f}$ Graph shows analysis of the percentage of genome edited efficiency in Tyr gene in WKY female $\mathbf{x}$ DA male (e) and WKY female $x$ male $(\mathbf{f})$ 
Table 2 Tyr-mediated mutations in F1 (WKY x DA) rat

\begin{tabular}{|c|c|c|c|c|c|c|}
\hline Stain & $\begin{array}{l}\text { Poring Pulse } \\
\text { Voltage }(V)\end{array}$ & Injected & Pregnant & Pups (A) & $\mathrm{KO}(\mathrm{B})$ & $\%(B / A)$ \\
\hline \multirow[t]{3}{*}{$\overline{\text { WKYO } \times \mathrm{DAO}^{\lambda}}$} & 50 & 10 & 9 & 46 & 27 & 58.7 \\
\hline & 40 & 10 & 9 & 68 & 24 & 35.3 \\
\hline & 30 & 9 & 8 & 67 & 9 & 13.4 \\
\hline \multirow[t]{3}{*}{ DA우 $\times$ WKY } & 50 & 9 & 6 & 19 & 8 & 42.1 \\
\hline & 40 & 9 & 5 & 26 & 10 & 38.5 \\
\hline & 30 & 7 & 6 & 28 & 4 & 14.3 \\
\hline
\end{tabular}

17.8\%, had indel mutation (knock-out; KO), and 26.9 and $11.1 \%$, had corrected alleles (knock-in; KI) at the target region, respectively (Table 3, Additional file 3: Table S4). Thus, these observations suggest that the rGONAD method is obviously feasible for gene correction in rat by the ssODN-based knock-in.

\section{Discussion}

The present study showed that the most suitable conditions for rGONAD method confirmed by electroporation efficiency. The rGONAD method allows efficient production of gene disrupted rats using CRISPR/Cas9 system. Furthermore, we demonstrated high efficiency of rGONAD method to produce knock-out and knock-in rats.

CRISPR/Cas9 system can be provided in genetically modified animals. However, it is difficult for the researchers to require high level technical expertise such as micromanipulation. Recently, electroporation-mediated in vitro transfection of isolated pre-implantation embryos has been reported in rats (TAKE methods) [26, 27]. Although this method is very convenient, it is limited only to bypass the microinjection step; the other two steps, isolation of embryos and embryo transfer could not be excluded.

On the contrary, the rGONAD method certainly avoided all the 3 major steps of producing the genomeediting rats. Thus, these features suggested that the rGONAD method is more reliable that does not require any specific skills and equipment such as micromanipulator and inverted microscope.

In our study, we were not able to obtain genome-editing rats using the rGONAD method in the super-ovulated female rats whereas the manipulated female rats became pregnant. This problem occurred in the super-ovulated female rats might be due to the following possibilities. In previous reports, super-ovulated female rats mated to males tended to low pregnancy rates though the number of blastocyst implantation in the super-ovulated females did not differ from those in normal rats [28, 29]. Similarly, our reports indicated that there were few pups in the superovulation with WKY and DA rats. On the other hand, we successfully demonstrated the rat rGONAD method without super-ovulated females. Moreover, we described a protocol for high efficiency for genome-editing using
rGONAD method as well as microinjection or in vitro electroporation. However, further studies are needed to draw a definite conclusion concerning super-ovulation and rGONAD method.

The laboratory rat has long been recognized as the preferred experimental animal in many areas of biomedical science [21]. In our previous study, anti-GBM nephritis can be induced in WKY rats by injection of monoclonal antibody against the NC1 domains of type IV collagen alpha chains [7, 8]. Although much progress has been made in understanding molecular mechanisms regulating anti-GBM nephritis, little is known about regarding molecular mechanism using gene disrupted rats.

In the present study, we showed high efficiency of the rGONAD method to produce knock-out and knock-in WKY rats. Taken together, using gene disrupted WKY rats as identified gene(s), the rat model of passively induced anti-GBM nephritis has tremendous potential for examining the mechanism by which inflammation is induced in human anti-GBM nephritis, as well as for identifying potential therapeutic targets. In addition in rats, there are several strains in order to perform specific studies, such as F344 for neurogenesis and SHR for hypertension [30]. In the future study, provided the genome-edited strain rats using the rGONAD method can be used in a variety of experiments.

In the recent study, it is certainly essential to meet with $3 \mathrm{R}$ principles of animal experimentation; Reduction, Replacement and Refinement, and further to understand welfare of animals [31]. The $i$-GONAD method does not require euthanasia of pregnant females, unlike in the traditional approaches where females need to be sacrificed for isolating zygotes for introduction of genome editing components ex vivo [24]. Moreover, we found that some female rats which underwent rGONAD method were able to deliver the pups (data not shown). These data indicate that the operated oviducts in the treated rats retained normal reproductive functions, pregnancy, and delivery. Finally, we strongly suggest that producing genomeediting animals with rGONAD method can be useful in variety of experimentation. 

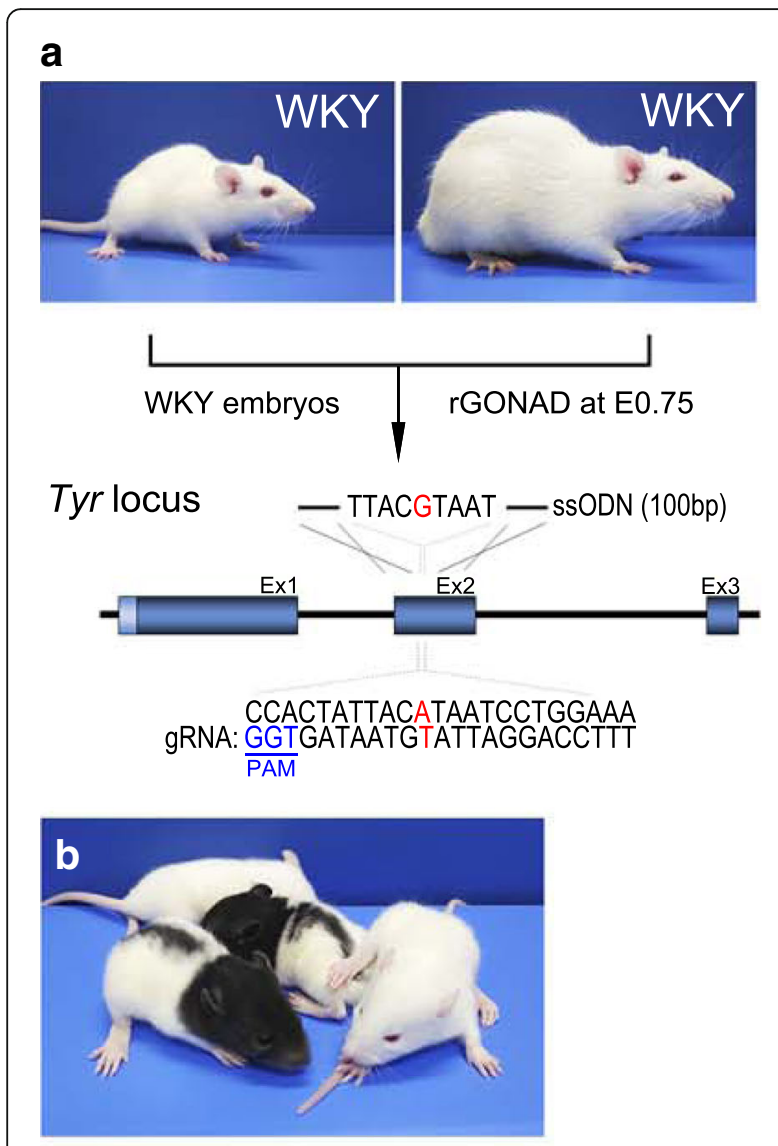

C

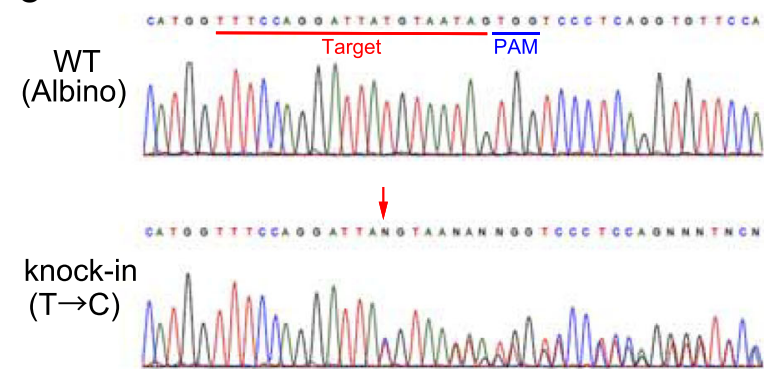

Fig. 3 Recovery of coat-color mutation in albino WKY rats using KI approach. a Scheme diagram of the recovery of coat-color mutation. The rGONAD method was performed in 0.75 day pregnant WKY female rats crossed to WKY male rats. The target sequence, PAM, and ssODN at Tyr locus are shown. $\mathbf{b}$ Picture of the knock-in rats showing the albino with non-agouti, hooded phenotype. c Direct sequencing results of wild-type WKY (upper; WT) or the editing (below; Knock-in) rats. Red arrow indicates that one allele had the corrected sequence ( $T$ to $C$ )

\section{Conclusions}

In conclusion, we established the method, namely rGONAD for generating genome editing rats. We confirm that the rGONAD method will facilitate the production of rat genome engineering experiments in many laboratories. Furthermore, rGONAD method can be readily applicable where traditional gene targeting using ES cells will not be well established in mammals such as guinea pig, hamster, cow, pig, and other mammals.

\section{Methods}

\section{Animals}

WKY/NCrl rats were obtained from Charles River, and DA/Slc rats were purchased from SLC Japan. The rats were kept with a 12:12-h light: dark cycle.

To investigate the electroporation efficiency (Fig. 1), rat females (WKY and DA) were super-ovulated by intraperitoneal injection of 20 IU pregnant mare serum gonadotropin (PMSG; ASKA Pharmaceutical Co. Ltd., Tokyo, Japan) and 10 IU human chorionic gonadotropin (hCG; ASKA Pharmaceutical Co. Ltd.). The super-ovulated females were mated to rat males (WKY and DA) overnight. To analyze CRISPR/Cas9 systems (see Figs. 2 and 3), estrous female rats were mated to male rats without superovulation. Presence of copulation plugs was confirmed by visual inspection in the following morning and used for the electroporation experiments.

All animals were handled in strict accordance with good animal practice as defined by the relevant national and/or local animal welfare bodies, and all animal works were approved by the appropriate committee.

\section{Preparation of fluorescence-labelled dextran}

To test the conditions of electroporation, we prepared 0 . $5 \mathrm{mg} / \mathrm{ml}$ Tetramethylrhodamine-labelled dextran (3 kDa; Thermo Fisher Scientific, Walttham, MA) and 0.1\% trypan blue (Nacalai tesque, Kyoto, Japan) diluted in OPTIMEM (Thermo Fisher Scientific).

\section{Preparation of CRISPR/Cas9 regents}

For the preparation of CRISPR/Cas9 regents, Alt- ${ }^{\mathrm{m}}$ CRISPR-Cas9 system (Integrated DNA Technologies [IDT, Coralville, IA]) was used in accordance with the manufacturer's protocol. Briefly, guide RNAs were designed using CHOPCHOP (http://chopchop.cbu.uib.no/). The synthetic crRNA and tracrRNA were commercially obtained as Alt$\mathrm{R}^{\mathrm{Tx}}$ CRISPR guide RNAs from IDT together with Cas9

Table 3 Coat-color phenotypes recovered from albino in WKY rat

\begin{tabular}{lllllll}
\hline Stain & $\begin{array}{l}\text { Poring Pulse } \\
\text { Voltage }(\mathrm{V})\end{array}$ & Injected & Pregnant & Pups (A) & KO (B) (\%: B/A) & KI (C) (\%: C/A) \\
\hline WKY $\times$ WKY & 50 & 8 & 6 & 26 & $13(50)$ & $7(26.9)$ \\
& 40 & 9 & 7 & 45 & $8(17.8)$ & $5(11.1)$ \\
\hline
\end{tabular}


protein (Alt-R ${ }^{\mathrm{mi}}$ S.p. Cas9 Nuclease 3NLS). Lyophilized crRNA and tracrRNA were resuspended in RNA-free Duplex Buffer to a concentration of $200 \mu \mathrm{M}$. Equal volume of crRNA and tracrRNA were mixed, and were heated at $95{ }^{\circ} \mathrm{C}$ for $5 \mathrm{~min}$. Then the mixture were removed from heat and placed at room temperature $\left(20-25^{\circ} \mathrm{C}\right)$ for more than an hour before the electroporation. ssODN donor (Additional file 3: Table S5) was custom synthesized from Eurofins Genomics Japan (Tokyo, Japan).

\section{rGONAD method}

$i$-GONAD method was performed as described previously [24]. Surgical procedures were operated on anesthetized female rats at Day 0.75 of pregnancy (E0.75: corresponding to late 1-cell stage zygotes; at 16:00 of the same day when plugs were confirmed) under observation using a dissecting microscope (SZX7; Olympus, Tokyo, Japan). Before injection, The annealed crRNA and tracrRNA were mixed with Cas9 (and ssODN) so that the final concentrations of components $30 \mu \mathrm{M}$ (for crRNA/tracrRNA), $1 \mathrm{mg} / \mathrm{ml}$ (for Cas9 protein), $1 \mu \mathrm{g} / \mu \mathrm{l}$ (for ssODN) diluted in OPTI-MEM and placed at room temperature $\left(20-25^{\circ} \mathrm{C}\right)$ for $5 \mathrm{~min}$. Approximately 2.-2.5 $\mu \mathrm{l}$ of electroporation solution (Tetramethylrhodamine-labelled dextran or CRISPR/Cas9 solution) was injected into the oviductal lumen from upstream of the ampulla using a micropipette. The micropipette apparatus consisted of a glass capillary needle (pulled using an electric puller: PN-31; Narishige, Tokyo, Japan). The electroporation was performed using a NEPA21 (NEPA GENE Co. Ltd., Chiba, Japan), generating two types of square pulses, namely poring and transfer pulses. The electroporation parameters were as follows; Poring pulse; 50 or 40 or $30 \mathrm{~V}, 5 \mathrm{msec}$ pulse, $50 \mathrm{msec}$ pulse interval, number of pulse 3 times, $10 \%$ decay ( \pm pulse orientation) and Transfer pulse; $10 \mathrm{~V}, 50 \mathrm{msec}$ pulse, $50 \mathrm{msec}$ pulse, number of pulse 3 or 6 times, $40 \%$ decay ( \pm pulse orientation) (see Fig. 1e, f). The rats were monitored for anesthesia recovery and were housed for further analysis.

\section{Genotyping}

Gene alterations were certified by PCR followed by DNA sequencing, as described previously [32]. Briefly, rat genomic DNA was isolated from the ear-piece or tail. PCR amplification was performed using the following primers. Direct sequencing was performed using the PCR products. PCR and sequence primers with the following primers: Tyr-fw (5'- ttggttttcacagatcatttg- $\left.3^{\prime}\right)$, Tyr-rv (5' - gctgaaattggcagttctatc- $\left.3^{\prime}\right)$.

\section{Fluorescent signal detection}

We collected the embryos at 2 days after electroporation. The embryos were observed using the fluorescence inverted microscope (Olympus IX73) for detecting the tetramethylrhodamine fluorescence.

\section{Additional files}

Additional file 1: Instillation of the solution containing tetramethylrhodamine-labelled dextran into oviductal lumen WKY female rats. (MP4 $2741 \mathrm{~kb}$ )

Additional file 2: Electroporation using a NEPA21 electroporator. (MP4 $6219 \mathrm{~kb})$

Additional file 3: Table S1. Tyr-mediated mutations in F1 (DA male/ WKY female) rat. Table S2. Tyr-mediated mutations in F1 (WKY male/DA female) rat. Table S3. Tyr-mediated mutations in F1 offspring. Table S4. Coat-color phenotypes recovered from albino in WKY rat. Table S5. CRISPR/Cas9 target sequence and ssODN used. (PDF $46 \mathrm{~kb}$ )

\section{Abbreviations}

Cas: CRISPR-associated; CRISPR: Clustered Regularly Interspaced Short Palindromic Repeats; GONAD: Genome editing via Oviductal Nucleic Acids Delivery; hCG: Human chorionic gonadotropin; i-GONAD: improved GONAD; PMSG: Pregnant mare serum gonadotropin; rGONAD: Rat improved GONAD; SSODN: Single stranded oligonucleotides; TALEN: Transcription activator-like effector nucleases; Tyr: Tyrosinase; WKY: Wistar Kyoto; ZFN: Zinc finger nucleases

\section{Acknowledgements}

We are grateful to Chieko Takahashi for technical assistance, and Dr. Tohru Okigaki for critical comments on the manuscript. We would like to thank Dr. Fumihiro Shigei, Chairman of the Board, Sowakai Medical Foundation, for financial support.

\section{Funding}

This work was supported in part by the Ryobi Teien Memory Foundation.

\section{Availability of data and materials}

All data during this study are included in this published article.

\section{Authors' contributions}

TK, MN, TK, MF, MS, MO, and MM conceived and designed the experiments, TK, MN, TK, and MM performed experiments, TK, MS, MO, and MM wrote the manuscript. All authors read and approved the final manuscript.

\section{Ethics approval and consent to participate}

All animals were handled in strict accordance with good animal practice as defined by the relevant national and/or local animal welfare bodies, and all animal works were approved by the Shigei Medical Research Institute Animal Care Committee (permission number: \#17007).

\section{Consent for publication}

Not applicable.

\section{Competing interests}

The authors declare they have no competing interests.

\section{Publisher's Note}

Springer Nature remains neutral with regard to jurisdictional claims in published maps and institutional affiliations.

\footnotetext{
Author details

'Division of Molecular Genetics, Shigei Medical Research Institute, 2117 Yamada, Minami-ku, Okayama 701-0202, Japan. ²Shigei Medical Research Hospital, Minami-ku, Okayama 701-0202, Japan. ${ }^{3}$ Section of Gene Expression Regulation, Frontier Science Research Center, Kagoshima University, Kagoshima, Kagoshima 890-8544, Japan. ${ }^{4}$ Department of Molecular Life Science, Division of Basic Medical Science and Molecular Medicine, Tokai University School of Medicine, Isehara, Kanagawa 259-1193, Japan. ${ }^{5}$ Center for Matrix Biology and Medicine, Graduate School of Medicine, Tokai University, Isehara, Kanagawa 259-1193, Japan. ${ }^{6}$ The Institute of Medical Sciences, Tokai University, Isehara, Kanagawa 259-1193, Japan.
} 
Received: 5 March 2018 Accepted: 20 March 2018

\section{Published online: 02 April 2018}

\section{References}

1. Atanur SS, Diaz AG, Maratou K, Sarkis A, Rotival M, Game L, Tschannen MR, Kaisaki PJ, Otto GW, Ma MC, et al. Genome sequencing reveals loci under artificial selection that underlie disease phenotypes in the laboratory rat. Cell. 2013;154:691-703.

2. Mullins LJ, Conway BR, Menzies RI, Denby L, Mullins JJ. Renal disease pathophysiology and treatment: contributions from the rat. Dis Model Mech. 2016:9:1419-33.

3. Chiang CK, Inagi R. Glomerular diseases: genetic causes and future therapeutics. Nat Rev Nephrol. 2010;6:539-54.

4. Yoshimi K, Mashimo T. Application of genome editing technologies in rats for human disease models. J Hum Genet. 2018;63(2):115-23. https://doi.org/ 10.1038/s10038-017-0346-2. Epub 2017 Nov 20.

5. Sado Y, Naito I, Akita M, Okigaki T. Strain specific responses of inbred rats on the severity of experimental autoimmune glomerulonephritis. J Clin Lab Immunol. 1986;19:193-9.

6. Aitman TJ, Dong R, Vyse TJ, Norsworthy PJ, Johnson MD, Smith J, Mangion J, Roberton-Lowe C, Marshall AJ, Petretto E, et al. Copy number polymorphism in Fcgr3 predisposes to glomerulonephritis in rats and humans. Nature. 2006;439:851-5.

7. Sado Y, Kagawa M, Rauf S, Naito I, Moritoh C, Okigaki T. Isologous monoclonal antibodies can induce anti-GBM glomerulonephritis in rats. J Pathol. 1992;168:221-7.

8. Sado Y, Boutaud A, Kagawa M, Naito I, Ninomiya Y, Hudson BG. Induction of anti-GBM nephritis in rats by recombinant alpha 3(IV)NC1 and alpha 4(IV)NC1 of type IV collagen. Kidney Int. 1998;53:664-71.

9. Capecchi MR. Gene targeting in mice: functional analysis of the mammalian genome for the twenty-first century. Nat Rev Genet. 2005;6:507-12.

10. Tong C, Li P, Wu NL, Yan Y, Ying QL. Production of p53 gene knockout rats by homologous recombination in embryonic stem cells. Nature. 2010:467:211-3.

11. Geurts AM, Cost GJ, Freyvert Y, Zeitler B, Miller JC, Choi VM, Jenkins SS, Wood A, Cui X, Meng X, et al. Knockout rats via embryo microinjection of zinc-finger nucleases. Science. 2009:325:433.

12. Cui $X$, Ji D, Fisher DA, Wu Y, Briner DM, Weinstein EJ. Targeted integration in rat and mouse embryos with zinc-finger nucleases. Nat Biotechnol. 2011;29:64-7.

13. Tesson L, Usal C, Menoret S, Leung E, Niles BJ, Remy S, Santiago Y, Vincent Al, Meng $X$, Zhang $L$, et al. Knockout rats generated by embryo microinjection of TALENs. Nat Biotechnol. 2011;29:695-6.

14. Hu X, Chang N, Wang X, Zhou F, Zhou X, Zhu X, Xiong JW. Heritable genetargeting with gRNA/Cas9 in rats. Cell Res. 2013;23:1322-5.

15. Li D, Qiu Z, Shao Y, Chen Y, Guan Y, Liu M, Li Y, Gao N, Wang L, Lu X, Zhao $Y$. Heritable gene targeting in the mouse and rat using a CRISPR-Cas system. Nat Biotechnol. 2013;31:681-3.

16. Li W, Teng F, Li T, Zhou Q. Simultaneous generation and germline transmission of multiple gene mutations in rat using CRISPR-Cas systems. Nat Biotechnol. 2013:31:684-6.

17. Harms DW, Quadros RM, Seruggia D, Ohtsuka M, Takahashi G, Montoliu L, Gurumurthy CB. Mouse genome editing using the CRISPR/Cas system. Curr Protoc Hum Genet. 2014;83(15 17):11-27.

18. Gurumurthy CB, Grati M, Ohtsuka M, Schilit SL, Quadros RM, Liu XZ. CRISPR: a versatile tool for both forward and reverse genetics research. Hum Genet. 2016;135:971-6.

19. Wang $H$, Yang $H$, Shivalila CS, Dawlaty MM, Cheng AW, Zhang F, Jaenisch R. One-step generation of mice carrying mutations in multiple genes by CRISPR/Cas-mediated genome engineering. Cell. 2013;153:910-8.

20. Yoshimi K, Kunihiro Y, Kaneko T, Nagahora H, Voigt B, Mashimo T. ssODNmediated knock-in with CRISPR-Cas for large genomic regions in zygotes. Nat Commun. 2016;7:10431.

21. Meek S, Mashimo T, Burdon T. From engineering to editing the rat genome. Mamm Genome. 2017;28:302-14

22. Takahashi G, Gurumurthy CB, Wada K, Miura H, Sato M, Ohtsuka M. GONAD: genome-editing via Oviductal nucleic acids delivery system: a novel microinjection independent genome engineering method in mice. Sci Rep. 2015:5:11406.

23. Gurumurthy CB, Takahashi G, Wada K, Miura H, Sato M, Ohtsuka M. GONAD: a novel CRISPR/Cas9 genome editing method that does not require ex vivo handling of embryos. Curr Protoc Hum Genet. 2016;88:Unit 1518.

24. Ohtsuka M, Sato M, Miura H, Takabayashi S, Matsuyama M, Koyano T, Arifin N, Nakamura S, Wada K, Gurumurthy CB. I-GONAD: a robust method for in situ germline genome engineering using CRISPR nucleases. Genome Biol. 2018;19:25.

25. Yoshimi K, Kaneko T, Voigt B, Mashimo T. Allele-specific genome editing and correction of disease-associated phenotypes in rats using the CRISPRCas platform. Nat Commun. 2014;5:4240.

26. Kaneko T, Sakuma T, Yamamoto T, Mashimo T. Simple knockout by electroporation of engineered endonucleases into intact rat embryos. Sci Rep. 2014;4:6382.

27. Kaneko T, Mashimo T. Simple genome editing of rodent intact embryos by electroporation. PLoS One. 2015;10:e0142755.

28. Zarrow MX, Clark JH, Roellig C, Denenberg VH. Prolonged gestation in the PMSG-treated immature rat. Biol Reprod. 1969;1:387-90.

29. Nuti KM, Sridharan BN, Meyer RK. Reproductive biology of PMSG-primed immature female rats. Biol Reprod. 1975;13:38-44.

30. Hunziker MH, Saldana RL, Neuringer A. Behavioral variability in SHR and WKY rats as a function of rearing environment and reinforcement contingency. J Exp Anal Behav. 1996;65:129-44.

31. Zhou Q. Balancing the welfare: the use of non-human primates in research. Trends Genet. 2014;30:476-8.

32. Matsuyama M, Nomori A, Nakakuni K, Shimono A, Fukushima M. Secreted frizzled-related protein 1 (Sfrp1) regulates the progression of renal fibrosis in a mouse model of obstructive nephropathy. J Biol Chem. 2014;289:31526-33.

\section{Submit your next manuscript to BioMed Central and we will help you at every step:}

- We accept pre-submission inquiries

- Our selector tool helps you to find the most relevant journal

- We provide round the clock customer support

- Convenient online submission

- Thorough peer review

- Inclusion in PubMed and all major indexing services

- Maximum visibility for your research

Submit your manuscript at www.biomedcentral.com/submit
Biomed Central 